Revue d'histoire de l'Amérique française

Q4. REVUE D'HISTOIRE DE L'AMÉRIQUE FRANÇAISE

\title{
Harmonie, écologie et nationalisme : la mise en parc de Forillon (1970-2012)
}

\section{Guillaume Blanc}

Volume 68, numéro 3-4, hiver-printemps 2015

URI : https://id.erudit.org/iderudit/1033641ar

DOI : https://doi.org/10.7202/1033641ar

Aller au sommaire du numéro

Éditeur(s)

Institut d'histoire de l'Amérique française

ISSN

0035-2357 (imprimé)

1492-1383 (numérique)

Découvrir la revue

Citer cet article

Blanc, G. (2015). Harmonie, écologie et nationalisme : la mise en parc de Forillon (1970-2012). Revue d'histoire de l'Amérique française, 68(3-4), 375-401. https://doi.org/10.7202/1033641ar
Résumé de l'article

L'histoire du parc national Forillon débute en 1970 par l'expropriation des populations et la destruction par le feu de leurs habitations. S'ensuivent alors quarante années de naturalisation de l'espace. Pelouses restaurées, montagnes reboisées et populations animales réintroduites deviennent les marqueurs d'un territoire désormais symbole de l'une des 39 régions naturelles définies par Parcs Canada, laquelle s'efforce, sans cesse, de faire voir et de faire croire à une nation naturellement canadienne. Cependant, ce récit naturel achoppe depuis peu sur l'impossibilité de concilier une territorialité locale présentiste et concrète avec une territorialité fédérale naturaliste et nationaliste vraisemblablement trop incohérente pour faire sens. 


\section{Harmonie, écologie et nationalisme : la mise en parc de Forillon (I970-20I2)}

Guillaume BlanC

Centre de recherches historiques/EHESS

RÉSUMÉ • L'histoire du parc national Forillon débute en 1970 par l'expropriation des populations et la destruction par le feu de leurs habitations. S'ensuivent alors quarante années de naturalisation de l'espace. Pelouses restaurées, montagnes reboisées et populations animales réintroduites deviennent les marqueurs d'un territoire désormais symbole de l'une des 39 régions naturelles définies par Parcs Canada, laquelle s'efforce, sans cesse, de faire voir et de faire croire à une nation naturellement canadienne. Cependant, ce récit naturel achoppe depuis peu sur l'impossibilité de concilier une territorialité locale présentiste et concrète avec une territorialité fédérale naturaliste et nationaliste vraisemblablement trop incohérente pour faire sens.

ABSTRACT - The story of the Forillon National Park began in 1970 with the expropriation of populations and the destruction by fire of their homes. The next forty years were devoted to the task of naturalizing the territory as lawns were restored, mountains reforested and animal populations reintroduced. These were the markers of a territory rebranded as one of the symbols of the 39 natural regions defined by Parks Canada, whose continuing intention was to present and make believable the notion of a naturally Canadian nation. However, this natural narrative recently got bogged down on the impossible task of merging a contemporary, concrete and local territoriality with a naturalist and nationalist federal territoriality seemingly too inconsistent to provide meaning.

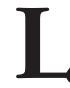

a mise en parc de la presqu'île de Forillon sur la péninsule gaspésienne (carte 1) renvoie, en 1970, à la place du Canada au Québec. L'administration québécoise exproprie les populations de Forillon, elle en 
cède la gestion à l'État fédéral puis, après avoir supervisé la destruction par le feu des habitations, les responsables de Parcs Canada définissent le thème du premier parc canadien au Québec: «l'harmonie entre l'homme, la terre et la $\operatorname{mer}^{1}{ }^{1}$. S'ensuit un façonnement de la nature dédié à l'édification d'un ordre social et naturel. Au fur et à mesure que les gestionnaires du parc donnent à voir le temps millénaire de la nature - celui de Parcs Canada et, par extension, de la nation canadienne -, ils donnent à croire en un temps de l'homme historique et révolu - celui de Forillon et, par extension, de la province de Québec. Cependant, depuis 2010, les responsables du parc entendent à la fois préserver l'«intégrité écologique» et l'intégrité culturelle» des lieux : le parc devrait être, et le «témoin authentique de la région naturelle des monts Notre-Dame et Mégantic», et l'incarnation «des valeurs culturelles eurocanadiennes de Forillon »' Cette réécriture du récit patrimonial suggère l'échec de l'écologie scientifique et de l'administration fédérale où elle s'exerce à publiciser un paysage national à même de transcender les territorialités provinciales et locales.

Le présent article s'intéresse à l'histoire de cette invention de la nature et de la nation canadiennes au Québec. Il retrace successivement quatre processus : le façonnement écologique d'un espace mis en parc, le façonnement symbolique d'un paysage mis en tourisme, le façonnement politique d'un territoire naturalisé pour être nationalisé et les aléas de la négociation économique et touristique sur laquelle semble achopper l'entreprise.

Rythmant la trame de notre récit, ces quatre éclairages successifs visent plusieurs objectifs. D’abord, éclairer la matérialisation puis la transmission paysagère d'une dualité entre l'histoire naturelle du Canada et l'histoire humaine du Québec permet d'envisager la nature comme instrument et révélateur de la construction nationale canadienne au Québec. Ensuite, notamment parce qu'il se découvre à travers l'emboîtement des documents produits à tous les échelons de l'action publique canadienne - fédérale, provinciale et locale -, le processus de naturalisation/canadianisation de l'espace-parc suggère, non pas l'intentionnalité d'une administration fédérale qui naturaliserait à dessein une portion du Québec, mais bien l'existence d'une culture canadienne selon laquelle la nature constitue un référent identitaire national. Enfin, sur place, l'analyse de la remise en

1. Ministère des Affaires indiennes et du Nord canadien (MAINC), Direction des parcs nationaux et des lieux historiques (DPN), Parc national Forillon. Plan-cadre provisoire, 1971, p. 3.

2. Parcs Canada (PC), Unité de gestion de la Gaspésie (UGC), Parc national du Canada Forillon. Plan directeur, 2010, p. 53. La Direction des parcs nationaux est rebaptisée Parcs Canada en 1973. 


\section{Carte I}

Forillon, parc national canadien au Québec

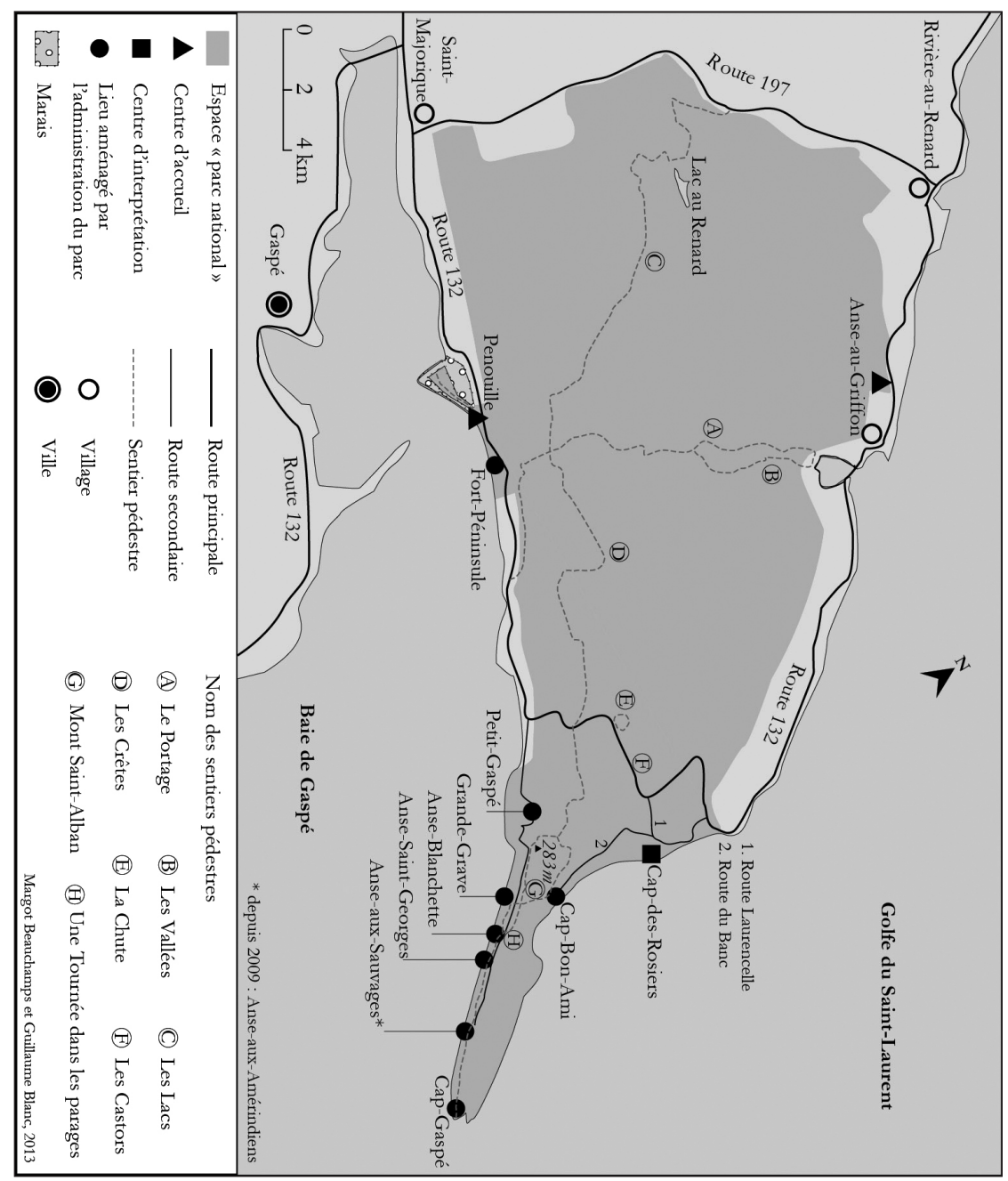

Carte réalisée à partir de: Parcs Canada, «Parc national Forillon. Carte du parc», www.pc.gc.ca/fra/ pn-np/qc/forillon/Information-aux-visiteurs/Cartes-Map.aspx (consulté le I3 avril 20I3). 
cause d'une nature atemporelle, an-anthropique et apolitique devrait contribuer à l'histoire environnementale des parcs nationaux canadiens. Bon nombre de travaux ont révélé l'intrication, au Canada, entre l'invention de la nature et la construction de la nation ${ }^{3}$. Moins nombreux, certains travaux ont également analysé le phénomène tel qu'il se développe au Québec, où les pouvoirs fédéraux recourent à l'argument économique ou scientifique pour mieux s'implanter dans la Province ${ }^{4}$. Inscrit dans les voies tracées par ces recherches, notre objectif est ici de retracer une histoire de la nation canadienne au Québec en rassemblant, en un seul récit, les dimensions performatives et coercitives de la nature et du pouvoir qui la façonne.

\section{FORILLON, PARC NATIONAL CANADIEN AU QUÉBEC}

Loin d'être révélatrice de celle du réseau des parcs nationaux du Canada, l'histoire ici retracée est celle d'un parc national canadien au Québec. En tant que tel, Forillon évolue au rythme d'un entremêlement d'échelles de pouvoir qu'il importe au préalable de décrire.

Tout d'abord, la création du parc est placée sous le signe d'une entente fédérale-provinciale destinée au développement du Bas-Saint-Laurent, de la Gaspésie et des Îles-de-la-Madeleine. En 1968, dans la foulée des travaux du Bureau d'aménagement de l'Est du Québec (BAEQ), le gouvernement québécois profite de la loi fédérale relative aux programmes économiques à frais partagés pour instaurer l'Office de développement de l'Est du Québec. Celui-ci prévoit l'aménagement d'un parc dans la presqu'île de Forillon, entreprise touristique censée pallier la déprise précipitée, au début du siècle, par le déclin de l'industrie de la pêche. Conformément à la «Loi concernant les parcs nationaux» de 1930, laquelle indique que la «terre [d'un parc] appartient à Sa Majesté», qu’elle doit être libre de droits et que seules les Provinces ont autorité pour acquérir les terres, l’Entente stipule que «le Québec se chargera de l'achat du terrain puis le cédera à

3. Voir notamment: Claire Elizabeth Campbell, dir., A Century of Parks Canada, 1911-2011 (Calgary, University of Calgary Press, 2011); Stéphane Héritier, «La nature et les pratiques de la nature dans les montagnes canadiennes: le cas des parcs nationaux de l'Ouest (Alberta et Colombie-Britannique)", Annales de géographie, 649, 3 (2005): 270-291; Alan MacEachern, Natural Selections. National Parks in Atlantic Canada, 1935-1970 (Montréal/Kingston, McGill-Queen’s University Press, 2001).

4. Aux travaux de Ronald Rudin et Jean-Marie Thibeault sur Forillon doit au moins être ajouté, sur le parc de la Mauricie: Olivier Craig-Dupont, «Hunting, Timber Harvesting, and Precambrian Beauties: The Scientific Reinterpretation of La Mauricie National Park’s Landscape History, 1969-1975 ", dans C. E. Campbell, dir., op. cit., 179-204. 
bail libre de charges ${ }^{5}$. Ainsi, le 17 juillet 1970, après avoir répertorié les terrains à exproprier, mis en œuvre un programme de relocalisation des populations puis transféré la propriété utile de Forillon à l'État fédéral, l'administration provinciale dépose devant l'Assemblée nationale la loi qui prive les habitants de leurs droits de résidence et d'exploitation ${ }^{6}$. En 1974, une fois les maisons brûlées et après une expropriation concernant «983 personnes, 225 familles, 214 propriétés résidentielles, 355 bâtiments, 1400 terres à bois, 8 fabriques et 5 municipalités ${ }^{7}$ », Forillon intègre la catégorie des parcs nationaux fédéraux ${ }^{8}$. Néanmoins, au lieu d'une cessation définitive des terres, non seulement le gouvernement provincial de Robert Bourassa obtient du gouvernement fédéral de Pierre Elliott Trudeau la signature d'un bail de 99 ans, mais il est aussi entendu que le Québec pourra reprendre possession du parc en l'an 2030, à condition de rembourser «le Canada de toutes les dépenses d'immobilisation encourues par ce dernier ${ }^{9} »$.

Aussi négociée soit-elle, cette prise de possession du territoire autorise ensuite la mise aux normes de l'espace-parc. S'agissant de la nature administrée, suivant les lignes directrices définies par Parcs Canada, le directeur du parc Forillon veille à ce que chaque "unité» - de conservation, d'accueil, d'interprétation et de recherche - concoure à la connaissance, l'aménagement et la protection des "ressources naturelles ${ }^{10}$ ». S'agissant de la nature réglementée, suivant les lois canadiennes sur la préservation «intacte» des parcs puis sur la préservation de leur "intégrité écologique $^{11} »$, des gardes patrouilleurs font respecter l'interdiction de la chasse, de la circulation automobile sur piste et ou encore de la pêche sportive

5. "Loi concernant les parcs nationaux", Statuts du Canada, $4^{\mathrm{e}}$ session du $16^{\mathrm{e}}$ Parlement chapitre 33 , 30 mai 1930, p. 281; ODEQ, Entente générale de coopération Canada-Québec. Le plan de développement du Bas Saint-Laurent, de la Gaspésie et des Îles de la Madeleine, Québec, 1968, p. 15.

6. "Loi concernant le parc Forillon et ses environs », Lois du Québec, chapitre 32, 17 juillet 1970, p. 159169. À propos des négociations entre les acteurs fédéraux, provinciaux et locaux, voir: Aryane Babin, L'expropriation du territoire de Forillon: étude du processus décisionnel des responsables étatiques fédéraux et provinciaux, 1968-1975, mémoire de maîtrise (histoire), Université Laval, 2013.

7. Jean-Marie Fallu, «La Gaspésie, cette éternelle région-pilote», Magazine Gaspésie, 47, 2 (2010): 10.

8. "Loi modifiant la Loi sur les parcs nationaux", Statuts du Canada, $29^{\mathrm{e}}$ législature $2^{\mathrm{e}}$ session chapitre 11, 7 mai 1974, p. 93-123.

9. Gouvernement du Québec, ministère des Affaires intergouvernementales, «Entente en vue des transferts de l'administration et du contrôle de terrains relatifs à l'aménagement d'un parc national dans la presqu'île de Forillon", Greffes des ententes intergouvernementales n¹970-12, 8 juin 1970, p. 2-4.

10. PC, Programme de Parcs Canada, 1975, p. 1; PC, Région du Québec (RQ), Parc national Forillon. Plan de conservation, 1981, p. 26-39.

11. "Loi concernant les parcs nationaux», op. cit., 1930, p. 282; "Loi modifiant la Loi sur les parcs nationaux et la Loi modifiant la Loi sur les parcs nationaux (sic)", Statuts du Canada, $33^{\mathrm{e}}$ législature $2^{\mathrm{e}}$ session chapitre 48, 18 août 1988, p. 1230. 
hors des zones autorisées ${ }^{12}$. S'agissant enfin de la nature circonscrite, Parcs Canada œuvre à la délimitation du contenant "parc national» - par l'édification de portes d'entrée au nord et au sud du parc - ainsi qu'à la classification écologique et à l'aménagement touristique de son contenu - par la division des $150 \mathrm{~km}^{2}$ du parc en zones de "préservation spéciale», de «milieu sauvage», de "milieu naturel» et de «loisirs», et par la construction de centres d'accueil et d'interprétation, d'aires de camping et d'activité, de sentiers et de belvédères ${ }^{13}$.

La mise en parc de Forillon témoigne donc autant du statut québécois de "société distincte» au sein de la Fédération que de la détermination fédérale à s'imposer au Québec. Contrairement à ce que suggère Ronald Rudin, Forillon n'est pas l'artefact exclusif du projet provincial conduit par les «nouveaux maîtres du Québec ${ }^{14}$ ». Parcs Canada y monopolise le contrôle de la nature et du citoyen et, comme en Mauricie où fut créé le second parc national canadien au Québec, Forillon évolue au gré d'une logique fédérale "descendante ${ }^{15}$ ». Les plans directeurs sont élaborés "par le parc national, le bureau régional et le bureau de Parcs Canada à Ottawa», et aujourd'hui encore, ils servent des "objectifs d'intérêt national $»^{16}$. Pour autant, contrairement à ce que suggère Jean-Marie Thibeault, Forillon n'est pas non plus un territoire exclusivement canadien, "cédé volontairement [...], pour la première fois de son histoire», par le Québec ${ }^{17}$. Signes de la présence québécoise, le «Centre de services du Québec - Parcs Canada» est officiellement responsable de l'aménagement du territoire, le directeur de Parcs Canada pour la «Région du Québec» nomme le directeur du parc Forillon, et celui-ci est toujours

12. MAINC, DPN, Compte-rendu des délibérations de l'audience publique tenue à Gaspé (Québec) le 17 novembre 1971, 1972, p. 24; MAINC, DPN, Parc national Forillon. Plan-cadre provisoire, 1971, p. 9; PC, RQ, Plan de gestion de la faune ichtyologique en milieu d'eau douce, 1979, p. 4.

13. PC, Parc national Forillon. Plan directeur, 1979, p. 5-13; PC, Parc national Forillon. Plan directeur, 1995, p. 39-68.

14. Ronald Rudin, "The 1st French-Canadian National Parks: Kouchibouguac and Forillon in History and Memory ", Journal of the Canadian Historical Association/Revue de la Société historique du Canada, 22, 1 (2011): 168 .

15. Olivier Craig-Dupont, Idéal de nature sauvage et transformation des territorialités au parc national de la Mauricie, 1969-1977, mémoire de maitrise (études québécoises), Université du Québec à Trois-Rivières, 2008, 118.

16. PC, Parc national Forillon. Plan directeur, 1979, p. 3 ; «Loi portant création de l’Agence Parcs Canada et apportant des modifications corrélatives à certaines lois ", Statuts du Canada, $36^{\mathrm{e}}$ législature $1^{\mathrm{re}}$ session chapitre 31, 3 décembre 1998, p. 1.

17. Jean-Marie Thibeault, La création d'un premier parc national au Québec. Le parc Forillon, 1960-1970, mémoire de maîtrise (histoire), Université de Sherbrooke, 1991, 2. 
sinon gaspésien, au moins québécois ${ }^{18}$. «Premier parc du réseau canadien en terre québécoise ${ }^{19}$ » et premier parc canadien à ne pas appartenir à la Couronne, Forillon évolue finalement au fil de la rencontre entre acteurs fédéraux, provinciaux et locaux.

Déterminés par cette dialectique du pouvoir, le façonnement écologique, symbolique et politique du parc Forillon, ainsi que sa remise en cause, éclairent la singularité historique d'un parc national canadien au Québec.

\section{UN FAÇONNEMENT NATIONAL DE LA NATURE}

Loin d'être le produit des milieux biogéophysiques tels qu'ils existaient avant leur mise en parc, la matérialité de l'espace-parc s'avère fonction d'un tri entre ce qui, dans la "nature», mérite ou non d'être préservé.

Tout au long des années 1970, les gestionnaires du parc procèdent à une rationalisation du territoire: ils inventorient les espèces, ainsi les mammifères marins, les localisent, ainsi les ongulés et ours noirs, puis les étudient, ainsi les cervidés et leurs "patrons d'utilisation du territoire ${ }^{20}$ ». Légitime parce que scientifique, l'administration restreint ensuite l'occupation de la presqu'île de Forillon: elle délimite des zones de protection autour des sites de reproduction des phoques, elle suspend la pêche sportive jusqu'à ce que la population de truites atteigne un effectif «conforme au but de conservation d'un parc», puis elle interdit toute forme d'exploitation forestière afin que se développe une végétation favorable à l'orignal ${ }^{21}$. Inversement, ses employés mènent des actions préventives, par exemple pour contenir la prolifération d'espèces telles que le porc-épic ou la tordeuse des bourgeons de l'épinette, celles-ci nuisant à la "protection des milieux $^{22}$ ». En 1986, Parcs Canada voit dans ces opérations la garantie de la "perpétuation d'un milieu naturel essentiellement non modifié par l'activité humaine». Et dix ans plus tard, les responsables du parc Forillon y voient un moyen d' "assurer l'évolution naturelle des ressources $»^{23}$.

18. Ces informations résultent du croisement des archives produites par les gestionnaires du parc depuis 1970.

19. PC, UGC, Parc national du Canada Forillon. Plan directeur, 2010, p. 4.

20. Section de conservation des ressources naturelles (SCRN), Parc national Forillon. Inventaire des mammifères marins, 1973, np. ; SCRN, Parc national Forillon. Inventaire de la neige au sol, 1974, p. 1; PC, RQ, SCRN, Mise à jour de l'inventaire des ongulés du parc national Forillon. 1982, 1986, p. 1.

21. PC, RQ, SCRN, Inventaire des mammifêres marins. Parc national Forillon, 1978, p. 94; «Lac au Renard: fermeture de la pêche», Le Pharillon, dans Centre de services du Québec - Parcs Canada (PC-CSQ), dossier "Revue de presse 3671-F/4», 22 juin 1982, n.p.; PC, Mise à jour de l'inventaire des ongulés du parc national Forillon. 1982, 1986, p. 47.

22. PC, Parc national Forillon. Plan de conservation, 1981, p. 33-37.

23. PC, RQ, SCRN, Activités et organisation du service de conservation du Parc national Forillon, 1986, p. 7 ; PC, RQ, SCRN, Parc national Forillon. Plan directeur, 1995, p. 12. 
Cependant, non seulement ces ressources étaient, avant 1970, le produit d'une interaction entre les hommes et leurs milieux, mais une fois patrimonialisées, elles continuent d'être façonnées par l'homme. Seulement, depuis, son action consiste à garantir la «constante progression» des espèces animales, la «régénération" des milieux humides, la "perpétuation» des espaces forestiers ${ }^{24}$, etc.

Cette dimension subjective de la sauvegarde de la nature se lit encore davantage à travers sa "réhabilitation». Prenons l'exemple des colonies de castors, au nombre de trois en 1971. L'arrêt de l'agriculture, de la foresterie et de la chasse favorise l'implantation de l'espèce au point qu'en 1986, le personnel de terrain recense 22 à 29 colonies. Certaines d'entre elles entrent toutefois «en conflit avec les valeurs du parc», leur établissement provoquant l'inondation de chemins et la déforestation d'espaces abritant une flore dite "menacée». Aussi, en 1993, Parcs Canada décide que le maintien des 73 colonies présentes doit être contrôlé. À cet effet, les agents du parc placent des tuyaux d'évacuation au fond de plusieurs étangs afin que leur niveau d'eau soit régulé, ils installent des clôtures et des piquets métalliques autour de barrages que les castors ne peuvent dès lors plus agrandir et, occasionnellement, ils capturent et relocalisent certaines colonies ${ }^{25}$.

Le traitement de cette espèce est loin de faire exception. D’une part, les « réintroductions » sont assez courantes. Ainsi, 27 faucons pèlerins sont acheminés en 1988 de l'Alberta à Forillon. Placés dans des cages artificielles, nourris de cailles et de poulets avant d'être libérés, ils sont, depuis, durablement installés dans le parc ${ }^{26}$. D’autre part, les espèces animales sont régulièrement délogées. C'est le cas d'une dizaine d'ours noirs capturés puis déplacés durant les années 1970 et $1980^{27}$. Ajoutée à la suspension du droit de chasse des cultivateurs et bûcherons, l'opération engendre une croissance exponentielle de leurs effectifs. Et si la direction du parc admet qu'il est contraire au principe d' «intégrité écologique pure» de suivre par télémétrie aérienne des ours munis de colliers émetteurs ou d'entretenir des corridors de déplacement par le biais de coupes forestières, elle n'en considère pas moins le résultat comme «identique à ce

24. Ibid., p. 12-25.

25. PC, RQ, SCRN, Étude sur le castor au parc national Forillon, 1987, p. 11 et 58; PC, Mise à jour des connaissances sur la population de castors du parc national de Forillon, 1993, p. 94-117.

26. PC, Suivi de la réintroduction du faucon pèlerin au parc national Forillon. Rapport d'opération, 1992, p. 1-8.

27. PC, Plan de gestion provisoire de l'ours noir ursus americanus au parc national Forillon, 1990, p. 1. 
qu'on observerait sous contrôle nature ${ }^{28}{ }^{\prime}$. En définitive, ce travail sur la nature consiste à en faire ce que l'on croit qu'elle fut, et ce que l'on veut qu'elle soit. Le déplacement des porcs-épics permet la régénérescence des pousses forestières recherchées par les ongulés, la fixation de quotas de pêche garantit la «reconstitution naturelle» de la faune ichtyologique et la plantation de sapins restaure «l'équilibre naturel» de la végétation ${ }^{29}$.

Ce façonnement du monde non humain trouve son pendant dans le monde humain. Comme le dira l'une des expropriés du parc, la nature y reprend sa place une fois "effacée toute trace de la sueur de [leurs] pères $^{30}$ ». Les maisons sont rasées et dix ans après, honorant les lignes directrices de la politique de protection de l'«intégrité écologique», les gestionnaires du parc évacuent vers le dépotoir de Gaspé les 135 tonnes de "déchets humains brûlés, enfouis, [...] oubliés ou inaperçus après l'expropriation ${ }^{31} »$. Sélectionnant ce qui, de l'anthropisation des lieux, doit être éliminé, les pouvoirs publics modèlent aussi ce qui, des temps et des espaces de l'homme, doit être protégé. Là encore, le protocole commence par la rationalisation de l'action à venir. L'administration fédérale étudie d'abord l'histoire des lieux: les passés amérindien, anglais et français, et les "genres de vie des pêcheurs-cultivateurs ${ }^{32}$ » qui leur succèdent. Elle identifie ensuite ce qui mérite attention: «le "mariage” de l’homme et de la nature " et la vie "traditionaliste» de "la période 1860-1880, soit un des moments où ces caractéristiques furent les plus prononcées ${ }^{33}$. Puis elle définit son objectif : «recréer le Forillon [...] de la fin du XIX ${ }^{e}$ siècle ${ }^{34}$ ».

Pour ce faire, les responsables du parc font du bâti le symbole d'un "paysage pittoresque ${ }^{35}$ ». À compter de 1978, ils entretiennent les quatre maisons encore sur pied dans «le respect de la tradition architecturale gaspésienne». En 1987, ils rénovent la chapelle Saint-Pierre de Petit-Gaspé, au nom de la «mixité religieuse» qui caractérisait Forillon. Puis en 1995,

28. Nathalie Leblanc et Jean Huot, Écologie de l'ours noir (Ursus americanus) au parc national Forillon. Rapport final présenté au Service de la conservation des écosystèmes (Ottawa, Parcs Canada, 2000), 96-100.

29. SCRN, Étude du porc-épic, 1979, p. 73; SCRN, Gestion de la faune ichtyologique. Rapport d'activité de la pêche sportive, 1986, p. 2 ; SCRN, Impact d'utilisation dans les secteurs d'aménagement du Parc Forillon, 1983, p. 1-55.

30. Blanche Fortin, citée par Sylvie Fortier, «Souvenirs d'expropriés», Gaspésie, 47, 2 (2010): 44.

31. PC, RQ, SCRN, Rapport d'activité. Élimination des dépotoirs familiaux. Parc national Forillon, 1983, p. 15.

32. PC, RQ, Parc national Forillon. Plan directeur, 1975, p. 22; DPN, Marcel Moussette, L'homme et son milieu naturel dans la région de Forillon, une esquisse ethnohistorique préliminaire, 1971, "Préface», n.p.

33. MAINC, Étude de la culture traditionnelle et de la géographie humaine du parc national Forillon, 1973, np.

34. DPN, Jean Simard, Inventaire des documents figurés (artefacts) du Parc National Forillon, 1971, p. 2.

35. Beauchemin-Beaton-Lapointe, Recherche du motif architectural. Rapport à la DPN, 1970, Gaspé, p. 1. 
ils restaurent les fortifications de Fort-Péninsule et le phare de Cap-Gaspé afin que ceux-ci "contribuent à l'appréciation du paysage culturel associé à l'occupation antérieure des lieux ${ }^{36}$ ». La direction du parc s'assure par ailleurs de l'entretien des havres de pêche. En 1980, après avoir démoli le quai de Petit-Gaspé jugé trop délabré, ses employés rénovent les havres de Cap-des-Rosiers et de Grande-Grave. Ils en préservent «le caractère traditionnel» et les maintiennent dans un état compatible avec «l'ambiance recherchée $»^{37}$. Enfin, quelques secteurs sont offerts à la mémoire agricole du territoire. Une fois la culture de la terre suspendue, la direction du parc décide de perpétuer «l'ouverture du paysage». Même en friches, les terres agricoles sont «maintenues» dix années durant comme "témoins de l'occupation humaine antérieure à la création du parc». Répartis entre Grande-Grave, la pointe Forillon et la vallée de l'Anse-au-Griffon, 86 hectares sont ensuite entretenus par le "brûlage», "la coupe à blanc», le "labourage», le «hersage» et le "contrôle chimique», cela afin d' «illustrer les dimensions des terres agricoles de l'époque et donner une impression de culture ${ }^{38} »$. En 2010, ce travail sur l'espace et le temps continue de faire perdurer «l'harmonie entre l'homme, la terre et la $\operatorname{mer}^{39} »$.

Le procédé participe à la muséification du territoire. Celle-ci est particulièrement frappante à Grande-Grave, au sud-est de la presqu'île. Dès 1971, les gestionnaires du parc entreprennent d'y restaurer le centre de pêche, l'entrepôt et les "maisons clés» où "réintégrer» les "documents œuvrés» produits par les populations autrefois résidentes ${ }^{40}$. Ils recréent un quotidien auquel ils ont eux-mêmes mis fin et au cours des années 1980, le site prend forme. Le magasin Hyman et l'établissement de l'AnseBlanchette sont emplis d' "artefacts du quotidien", les deux bâtiments accolés au havre de pêche sont rénovés et les dépendances encore debout «mises en valeur » ${ }^{41}$. Considérant Grande-Grave comme «le secteur le plus à même d'expliciter le thème du parc », Parcs Canada y réhabilite alors, durant les années 1990, d'anciens espaces agricoles et une vingtaine de bâtiments usuels. Elle œuvre de la sorte au «maintien du caractère propre à ce lieu $»^{42}$.

36. PC, Parc national Forillon. Plan directeur, 1979, p. 12; Environnement Canada. Parcs, Forillon, 1987, p. 36; PC, Parc national Forillon. Plan directeur, 1995, p. 45-46.

37. PC, RQ, Plan de service. Les Havres et quais du parc national Forillon, 1980, np.

38. PC, RQ, SCRN, Plan de gestion des terres agricoles en friche. Parc national Forillon, 1983, p. 1 et 60-74.

39. PC, UGC, Parc national du Canada Forillon. Plan directeur, 2010, p. 52.

40. DPN, Jean Simard, Inventaire des documents figurés (artefacts) du Parc National Forillon, 1971, p. 5.

41. PC, Pierre Drouin, La maison Hyman à Grande-Grave, parc national Forillon, 1984, p. 4; PC, Amendement au plan de gestion. Parc national Forillon, 1988, p. 10.

42. PC, RQ, Programme de collection, 1994, p. 6; PC, Parc national Forillon. Plan directeur, 1995, p. 45-46. 
Ce processus de destruction/valorisation des signes de l'occupation humaine distingue Forillon des parcs canadiens où l'administration fédérale a effacé toute trace attestant d'une anthropisation passée. Mais Forillon est lui aussi de ces lieux qui sont naturels parce qu'une culture les a faits et déclarés «naturels ${ }^{43}$ ». Au fur et à mesure qu'elle donne à croire en un lien matériellement révolu entre l'homme et la terre, sa matérialité donne à voir un paysage où le temps de l'homme se serait harmonieusement estompé derrière celui de la nature.

\section{UNE ÉNONCIATION NATURELLE DE LA NATION}

Écosystème et paysage, Forillon doit susciter un sentiment vis-à-vis de la nature et de la nation. Ses gestionnaires influencent pour cela la lecture de l'espace que découvrent chaque année, depuis 1970, 80000 à 140000 visiteurs $^{44}$. Par le biais d'expositions, de publications et d'animations, ces derniers se voient invariablement présenter le même paysage. Au début des années 1980 par exemple, en assistant aux «causeries » proposées par les centres d'information du parc, en lisant le guide touristique officiel de Forillon ou en assistant à l'exposition abritée par l'ancien magasin Hyman, un visiteur peut "découvrir le mode de vie passé des habitants», «ressentir [...] l'intensité des vies humaines qui se vécurent dans un contexte naturel» ou encore, appréhender «les rapports éco-culturels qui existent entre le milieu humain et le milieu naturel $»^{45}$. Cette offre discursive d'un paysage un jour culturel mais toujours naturel est complétée par la promotion d'un certain usage du territoire. Voyant dans ses parcs des "sanctuaires de nature et de solitude où l'homme peut trouver la "re-création" du corps et de l'esprit», Parcs Canada prévoit y combiner en 1969 "conservation" et «re/é-création $»^{46}$. Dans cette perspective, à Forillon, ses employés font de la route un outil privilégié d'appréhension de la nature. De 1970 à 1975, ils réhabilitent le tronçon routier de Penouille au sud, rénovent à l'est le chemin Laurencelle et la route du Banc et ferment, au centre et à l'est de la presqu'île, les routes du Portage et de Cap Gaspé.

43. A. MacEachern, op. cit., 4.

44. PC, Éléments d'informations. Parc national Forillon, 1973, p. 8; PC, UGC, Parc national du Canada Forillon. Plan directeur, 2010, p. 25.

45. PC, Cahier spécial «Le parc national Forillon», juillet 1981, p. 1-2; PC, Maxime St-Amour, Guide du parc national Forillon. L'harmonie entre l'homme, la terre et la mer (Vancouver/Toronto, Douglas \& McIntyre, 1984), 83 ; PC, RQ, Répertoire des artefacts historiques de l'exposition "Au rythme des saisons", 1989, 7.

46. J. I. Nicol (Directeur des parcs nationaux et historiques), "The National Parks Movement in Canada», dans J. G. Nelson et R. C. Scace, dir., Canadian Parks in Perspective (Montréal, Harvest House, 1970), 20. J. I. Nicol évoque dans ce texte rédigé en anglais la «recreation» et la «re-creation». 
Dès lors, sur la route et les belvédères construits à son intention, seul un paysage englobant systématiquement «la terre et la $\mathrm{mer}^{47}$ » se présente au visiteur.

Dans le reste du parc, l'accessibilité aux lieux de la nature lui est garantie. Dès les années 1970, les responsables du parc aménagent des "terrains de camping et de pique-nique, des promenades panoramiques, des sentiers pour les randonnées à pied ou à cheval, des postes d'observation [...], des endroits de repos, des installations pour le bain et la pratique des sports ${ }^{48}$. Au fil du temps, ce dispositif d'accueil est agrandi et réparti au sein de 11 «aires de récréation» et «d'interprétation». Son objectif reste toutefois identique. Dès ses débuts, l'espace-parc est dédié à l'offre publique, sur la côte septentrionale, de la "géologie de la presqu'île [...] avec le témoignage d'un temps passé où l'homme devait s'adapter à un milieu austère», sur les massifs de l'ouest, de «la tranquillité de la beauté sauvage» et au cœur du parc, de "la nature sauvage et solitaire ${ }^{49}$. Représentant comme les autres parcs du pays le "visage originel du Canada », Forillon donnerait ainsi à la communauté nationale un moyen de se récréer, voire de se re-créer.

Instruments d'une éducation du regard, ces «mécanismes de fabrication et d'entretien ${ }^{50}$ » de l'espace-parc servent à publiciser l'histoire humaine et révolue d'une nature intacte et atemporelle. Une nation ne peut exister sans référence qui rende son peuple "présent à l'histoire ${ }^{51}$ » et c'est pourquoi, à Forillon comme dans les autres parcs du Canada ${ }^{52}$, l'administration fédérale s'efforce d'agréger la communauté nationale autour d'une référence paysagère qui lui parle autant de son passé que de son présent et, celle-ci étant conservée "à perpétuité53 ", de son avenir.

L'entreprise nécessite en premier lieu d'inscrire dans le domaine public une nature sublime et authentique. Grâce à la réhabilitation de chemins pédestres et à l'organisation de croisières en haute mer dans les années 1970, à l'aménagement de points de vue sur les anciennes terres agricoles et de sentiers d'hiver dans les années 1980, puis à l'entretien des lacs d'eau douce et à la construction de belvédères orientés vers des espaces repeu-

47. MAIN, DPN, Parc national Forillon. Plan-cadre provisoire, 1971, p. 16.

48. Forillon, Québec, Entente générale de coopération Canada-Québec, 1970, p. 12.

49. PC, RQ, Plan d'interprétation. Parc national Forillon, 1976, p. 17.

50. Philippe Descola, «Postface. Les coulisses de la nature», dans Adel Selmi et Vincent Hirtzel, dir., Gouverner la nature (Paris, Éditions de l’Herne, 2007), 124.

51. Fernand Dumont, Genèse de la société québécoise (Montréal, Les Éditions du Boréal, 1993), 236.

52. Stéphane Héritier, "Une Nature monumentale. Paysage et patrimoine dans les Alpes canadiennes",M@ppemonde, 104, 4 (2011).

53. MAINC, DPN, Politique des parcs nationaux, 1969, p. 2-5. 
plés en faune dans les années 1990, Parcs Canada met à la disposition du public des paysages «de carte postale», «d'une beauté exceptionnelle», voire "grandioses $\|^{54}$. Cette contemplation et cette pratique paysagères doivent susciter un sentiment d'expérience de la nature. En 1971, l'administration entend faire de la marche à pied un outil par lequel «procurer à chacun une expérience individuelle plus intense», et dix ans plus tard, elle considère la pêche comme un moyen "de "sentir" " la nature. Depuis, le discours est élargi à l'ensemble de l'espace-parc. Parcs Canada y propose en 1995 «la qualité de l'expérience authentique» puis, en 2010, une interaction avec la nature qui "éveille les sens, suscite des émotions " ${ }^{55}$. Systématiquement, l'imaginaire mobilisé est celui d'une nature intacte qui suggère, le patrimoine aidant, un sentiment de permanence.

Faire du parc le symbole de la permanence de la nature et de la nation qui le préserve nécessite, en second lieu, de faire croire à un temps humain suranné. «Royaume des pêcheurs saisonniers» au XVIII siècle, territoire relativement intégré à l'industrie internationale de la pêche au XIX ${ }^{\mathrm{e}}$ puis espace agro-forestier-maritime en croissance au début du XX ${ }^{\mathrm{e} 5}$, la Gaspésie orientale est le lieu de la déprise et de l'aide publique depuis la fin de la Première Guerre mondiale ${ }^{57}$. Les gestionnaires du parc Forillon font cependant peu de cas de cette histoire récente. Écartant dans le discours et dans l'espace les marques de ces "changements socio-économiques», ils se concentrent exclusivement sur le "profil de Grande-Grave au XIX ${ }^{e}$ siècle $^{58}$ ». En 1976, après avoir retiré les enseignes publicitaires sur l'ancien magasin Hyman et fabriqué un treillis de branchage de sapin sur les terrains alentours, ils inaugurent le long du littoral un «sentier pittoresque » dédié, sur trois kilomètres, à l’«ambiance naturelle authentique» de «la vie d'autrefois». Des «îlots» informatifs sont répartis trois ans plus tard le long du sentier. Ils rappellent l'importance des compagnies de pêche et la

54. Forillon, Québec, Entente générale de coopération Canada-Québec, 1970, p. 1; PC, Parc national Forillon. Plan de mise en valeur de la zone marine, 1980, p. 13; PC, Parc national Forillon. Guide des activités récréatives et d'interprétation, 1996, p. 3.

55. MAINC, DPN, Parc national de Forillon, concept du parc, 1971, np; PC, RQ, Plan de service. Les Havres et quais du parc national Forillon, 1980, np.; PC, Parc national Forillon. Plan directeur, 1995, p. 35 ; PC, UGC, Parc national du Canada Forillon. Plan directeur, 2010, p. 2.

56. Jules Bélanger, Marc Desjardins et Yves Frenette, avec la collaboration de Pierre Dansereau, Histoire de la Gaspésie (Montréal, Boréal Express / Institut québécois de recherche sur la culture, 1981), 99, $139,441$.

57. Maryse Grandbois, «Le développement des disparités régionales en Gaspésie 1760-1960», Revue d'histoire de l'Amérique française, 36, 4 (1983): 500-506.

58. PC, Diane Turcotte, Analyse et synthèse des éléments pertinents pour l'interprétation, 1974, p. 15. 
centralité du havre pour la "vie communautaire», le tout dans une constante «harmonie» avec la terre et la mer.

Puis, au milieu des années 1980, là où «l'ameublement d'époque» des deux anciennes habitations de l'Anse-Blanchette présente au public «le milieu naturel dans lequel ont vécu la plupart des Gaspésiens ${ }^{59}$ ", les charrettes et traineaux de chasse disposés le long du sentier rebaptisé "Une tournée dans les parages" servent «de fond de scène au paysage ${ }^{60}$ ». L’administration véhicule enfin explicitement, depuis 1990, la représentation d'un territoire autrefois historique, reliquat «de la mémoire de l'aventure gaspésienne $^{61}$ ». Aussi, l'homme n'est plus au cœur du paysage. Ses traces sont valorisées uniquement pour être mieux dépassées, et seule la nature demeure dans ce territoire où l'homme s'est contenté de vivre, un temps, en harmonie avec la nature qui le précédait et qui lui a survécu.

À terme, Forillon favorise l'articulation du territoire local au territoire national. En une journée, il est possible de parcourir la plage de Penouille, de poursuivre sa route jusqu'au mont Saint-Alban et, après une ascension suivie d'une pause déjeuner à Grande-Grave, de se promener sur le sentier longeant, toujours au sud de la péninsule, la pointe de Forillon.

À partir des belvédères aménagés sur les hauteurs du mont Saint-Alban ou sur le plateau de l'Anse-Saint-Georges, le visiteur est amené à poser son regard sur l'espace. Envisagé comme sublime, mélancolique ou morne, le paysage suscite d'abord l'émotion esthétique. Les promenades effectuées le long de Penouille et de la pointe de Forillon évoquent ensuite la profondeur historique du territoire. Grâce à un panneau informatif situé entre le parking et le début de la plage, le public apprend que c'est probablement ici, à Penouille, que Jacques Cartier a accosté en 1534 et établi le contact avec les Amérindiens micmac. Quelques kilomètres plus loin, au départ d'un sentier débutant à Grande-Grave, un autre panneau indique au visiteur que les maisons vers lesquelles il se dirige sont celles de l'Anse-Blanchette où, entre la fin du XVIII ${ }^{e}$ siècle et le début du XX ${ }^{\mathrm{e}}$ siècle, quelques familles vécurent d'une pêche traditionnelle et d'une activité agro-forestière d'autosubsistance. Objets de mémoire pour cer-

59. PC, Rapport de synthèse. Projet d'interprétation historique (provisoire) à Grande-Grave, 1976, p. 4-7; PC, Grande-Grave, programme d'exposition, 1979, p. 2-5 ; PC, Parc National Forillon, Anse-Blanchette, 1984, p. 17.

60. PC, Rapport de synthèse. Projet d'interprétation historique (provisoire) à Grande-Grave, 1976, p. 4-7; PC, Grande-Grave, programme d'exposition, 1979, p. 2-5 ; PC, Parc National Forillon, Anse-Blanchette, 1984, p. 17; PC, Amendement au plan de gestion. Parc national Forillon, 1988, p. 36.

61. PC, Concept de mise en valeur. Maison Dolbel-Roberts, Parc national du Canada Forillon, 2004, p. 7. 
tains, d'autres lieux sont enfin sublimés par leur allure foncièrement naturelle.

Comme le révèlent les photographies de Cap-des-Rosiers, l'administration ne se contente pas d'enraciner l'espace-parc dans un temps long de quelques siècles. À la suite de l'élimination de la présence humaine dont seul un visiteur informé aura connaissance, le paysage semble naturel, c'est-à-dire tout simplement là, sans qu'il y ait quoi que ce soit à changer et sans que quoi que ce soit ait l'air d'y avoir changé depuis un temps indéfinissable. Plus qu'ancré dans l'histoire, le territoire apparaît hors de l'histoire.

Ces sentiments - l'esthétique, l'histoire et la transcendance du temps historique - sont le produit d'un investissement nostalgique du territoire, condition de l'inscription des hommes dans l'espace social en général, et dans l'espace national en particulier ${ }^{62}$. Antérieure à «l'avènement de la société agricole puis de la société industrielle » à partir desquelles «l'environnement s'est dégradé », la mémoire du territoire est celle d'une nation naturelle, produit d'un passé durant lequel, «pendant des millions d'années, les forces de la nature et non les activités de l'homme, ont agi»" ${ }^{63}$. Dispositif nostalgique et discursif suffisamment puissant pour se passer de mots, Forillon peut ainsi susciter chez les individus cette «sorte de connivence mémorielle» par laquelle leur inscription dans le paysage local s'étend naturellement au territoire national qu'il symbolise ${ }^{64}$. Forillon peut également susciter une forme d'affection paysagère, "sous-entendant forcément une extension du local au national: de l'amour d'un petit territoire à l'amour d'un territoire plus vaste ${ }^{65}$ ».

\section{PRODUCTION DE LA NATURE ET PROTECTION DE LA NATION}

Fidèle au principe selon lequel pour les hommes, le «sens du réel dépend entièrement de l'apparence, et donc de l'existence d'un domaine public ${ }^{66}$ ", l'État fédéral use de la nature pour concrétiser les contours matériels et idéels de la nation. Protégée pour être consommée, cette nature vise à pallier un passé manquant de profondeur et débordant, quoique relativement comparé par exemple à son voisin du Sud, de conflits.

62. Philippe Gervais-Lambony, Territoires citadins. 4 villes africaines (Paris, Belin, 2003), 140.

63. PC, Principes directeurs et politiques de gestion, 1994, p. 24.

64. François Walter, «Les échelles d'un imaginaire paysager européen dans l’histoire», dans Mario Bédard, dir., Le paysage. Un projet politique (Québec, Presses de l’Université du Québec, 2009), 60.

65. François Walter, Les figures paysagères de la nation. Territoire et paysage en Europe $\left(16^{e}-20^{e}\right.$ siècles) (Paris, EHESS, 2004), 178.

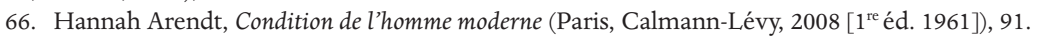




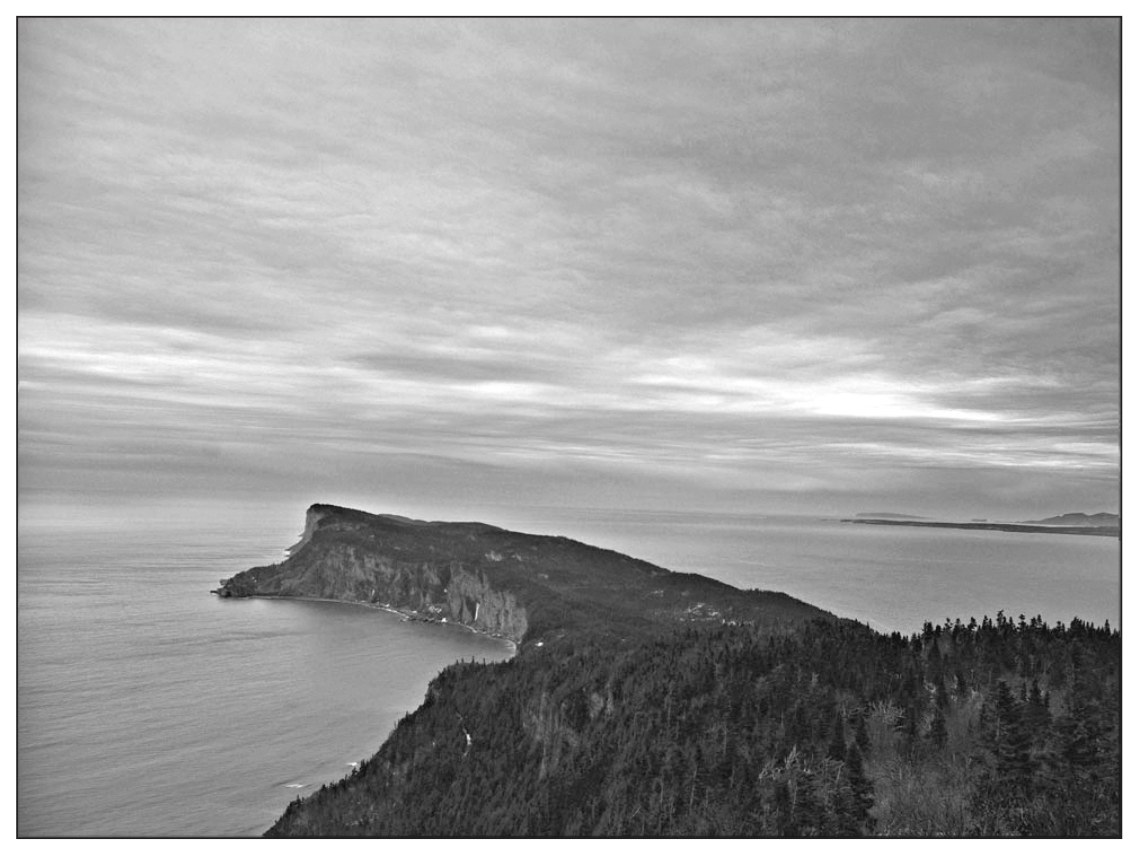

Cliché de l'auteur, vue du mont Saint-Alban, avril 2012. 个

Cliché de l'auteur, Anse-Saint-Georges, avril 2012.

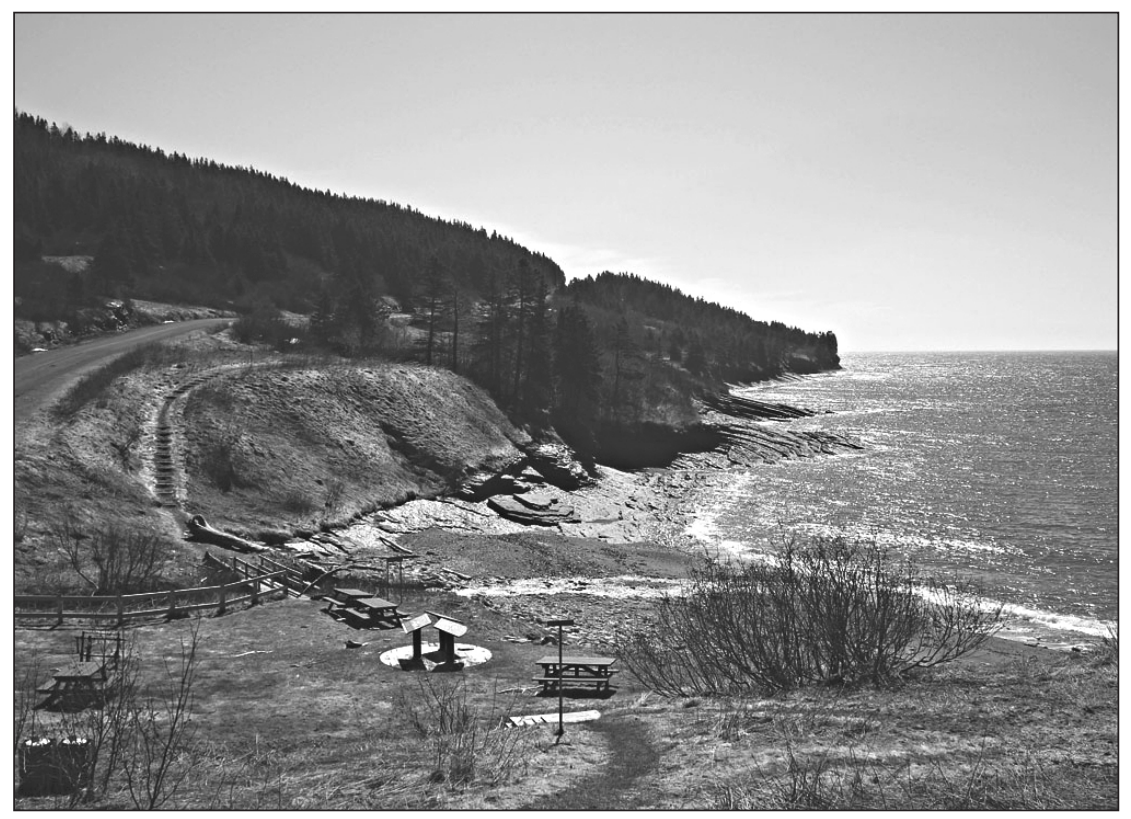




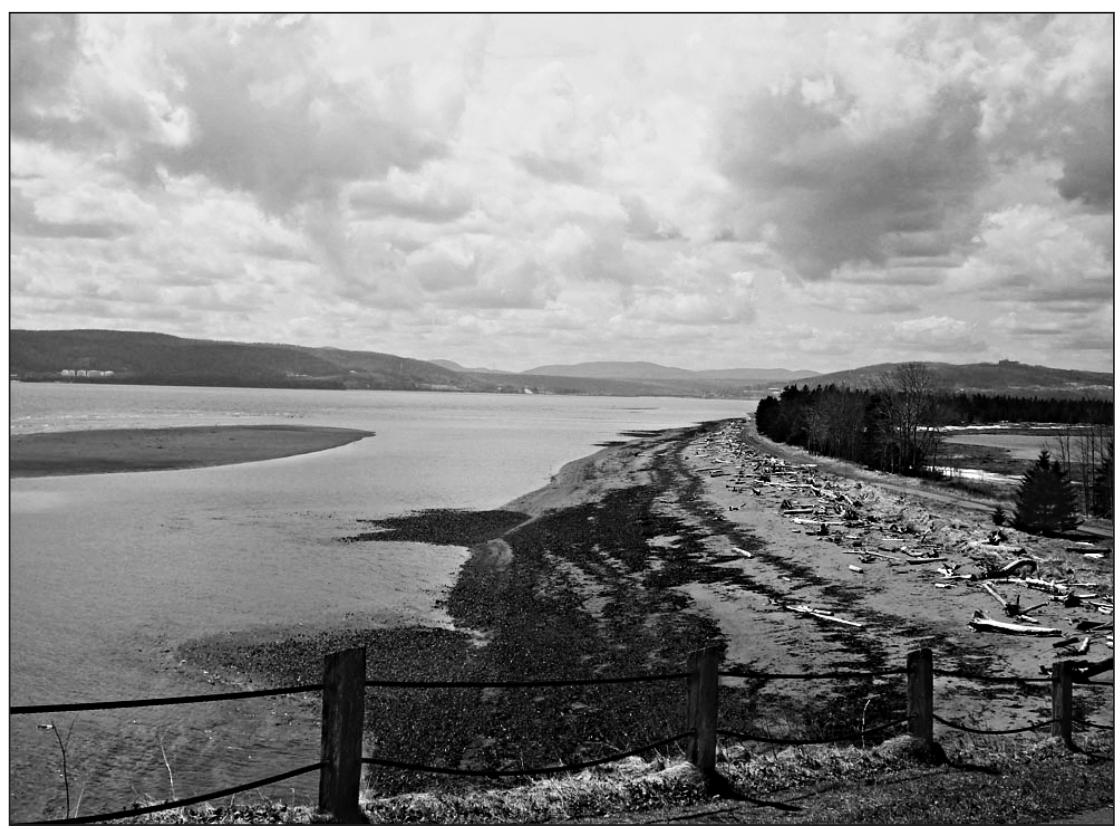

Cliché de l'auteur, Penouille, avril 2012. 个

Cliché de l'auteur, Anse-Blanchette, avril 20I2. $\quad \downarrow$

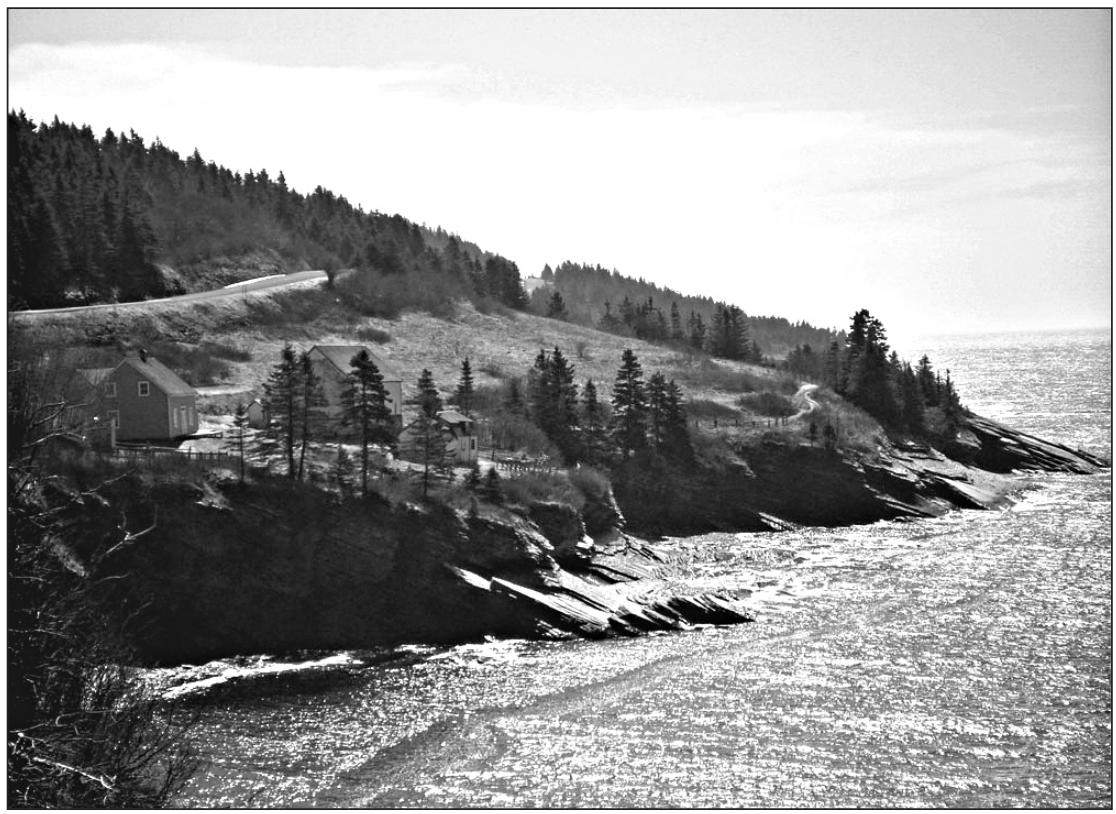




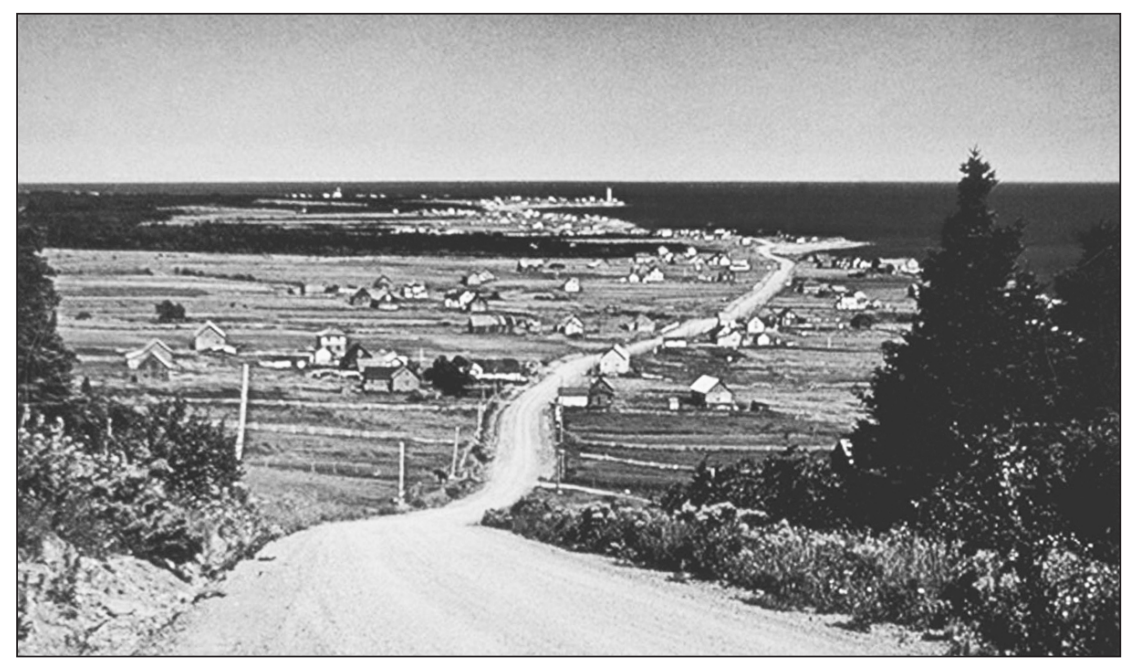

Cliché de la famille Kavanagh, Cap-des-Rosiers, pré-1970.

Cliché de l'auteur, Cap-des-Rosiers, mai 2012. $\downarrow$

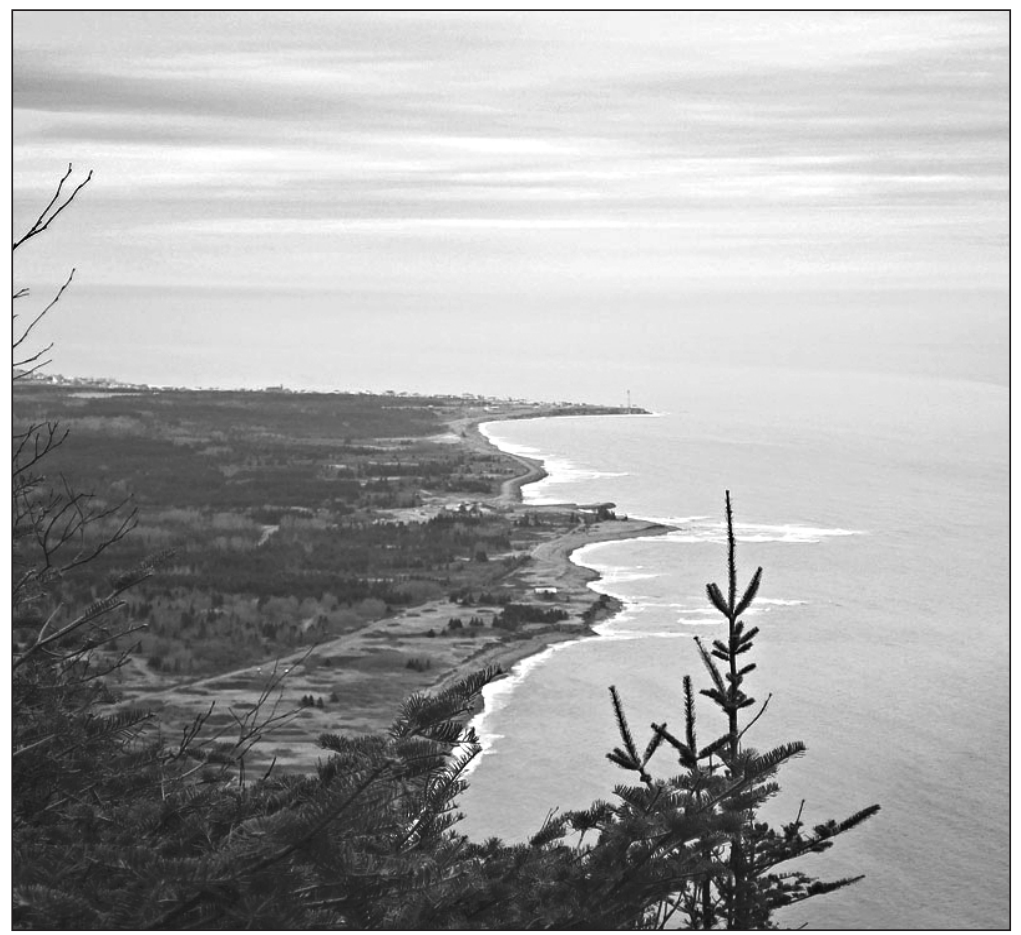

www.pages.infinit.net/kagh/origine.htm (consulté le 8 janvier 20I3). 
Le processus débute au début du XX $\mathrm{XX}^{\mathrm{e}}$ siècle. Créée en 1919 pour désigner les sites nationaux à commémorer, la Commission des lieux et monuments historiques du Canada fait face aux provinces de l'Ouest et de l'Est attentives à l'histoire régionale et, d'un autre côté, au Québec et à l'Ontario qui s'opposent à propos des sites représentatifs de la «nation". Contraignant la Commission à fabriquer un patrimoine national dénué d'idéologie nationaliste ${ }^{67}$, ces velléités conduisent l'État fédéral à se tourner vers la wilderness, "médium» à travers lequel transcender les mémoires concurrentielles ${ }^{68}$. Jusqu’à présent instaurés au cas par cas, les parcs nationaux sont mis au service de cette politique de la nature. La création de parcs dans les montagnes Rocheuses et en ColombieBritannique favorise l'incorporation de l'Ouest dans la communauté imaginée canadienne et en 1930, la «Loi concernant les parcs nationaux» encourage la création de parcs dans l'ensemble de la Confédération ${ }^{69}$. Parce qu'ils réclament le droit à l'enseigne "parc national» mais qu'ils rechignent à racheter la propriété éminente des terres pour la céder à l'administration fédérale, les gouvernements provinciaux ralentissent d'abord l'entreprise ${ }^{70}$. En revanche, au tournant des années 1960, l'essor du tourisme et la mise en place de programmes fédéraux à frais partagés l'emportent sur les réserves économiques et politiques des provinces. Il faut dire que le pouvoir fédéral redouble alors d'efforts. Dans la droite ligne de la "canadianisation» du pays au cours des années 1950 et de la «réification nationale» des années $1960^{71}$, l'État jette les bases, en 1969, d'un "réseau de parcs nationaux» représentatifs des "beautés naturelles du Canada», ces «sources d'inspiration créatrice» dont «les Canadiens devraient profiter le plus possible dans l'intérêt même de l'épanouissement culturel du pays ${ }^{72}$ ».

En 1970, la création du parc Forillon s'inscrit dans ce contexte fédéral de construction nationale. En troquant la cessation immuable de la presqu'île contre un bail emphytéotique, le gouvernement de Bourassa

67. Juliette Dutour, La construction du patrimoine du Canada entre reconnaissance publique et valorisation touristique, thèse de doctorat (histoire), Université Laval/EHESS, 2009, 47-73.

68. George Altmeyer, "Three ideas of Nature in Canada, 1893-1914», dans Chad et Pam Gaffield, dir., Consuming Canada. Readings in Environmental History (Toronto, Clark, 1995), 113.

69. Alisa Catharine Apostle, The View from the Hill. National Park Culture and Gatineau Park, 1920-1960, mémoire de maîtrise (arts), Queen's University, 1997, 33.

70. Lloyd Brooks, «Planning a Canadian National Park System - Progress and Problems », dans J. G. Nelson et R. C. Scace, dir., Canadian Parks..., op. cit., 316.

71. Ryan Edwardson, Canadian Content. Culture and the Quest for Nationhood (Toronto, University of Toronto Press, 2008), 16 ("Canadianization», "national reification»).

72. MAINC, DPN, Politique des parcs nationaux, 1969, p. 3, 14. 
se veut le digne héritier de la Révolution tranquille. Il impose à la fédération une nouvelle preuve du statut sinon national, au moins distinct, du Québec. Ce "province-building» n’entame en rien, cependant, le «nationbuilding ${ }^{73}$ défendu par le gouvernement canadien de Trudeau. Tandis que Jean Chrétien, ministre des Affaires indiennes et du Nord canadien, se félicite qu'il y ait «maintenant un parc national dans au moins chaque province $^{74}$ », la Direction des parcs nationaux voit dans la naissance de Forillon, sur la pointe atlantique du Canada, nouvelle traduction matérielle de la devise nationale $A$ mari usque ad mare ma $^{75}$ la complétion d'un réseau de parcs désormais «exactement représentatif du pays ${ }^{76}$ ».

Ce planter de drapeau en terre québécoise est rapidement soutenu, à Forillon, par l'effacement des traces récentes de la présence humaine et à l'échelle nationale, par une véritable "naturalisation de la nation ${ }^{77}$ ». L'administration fédérale théorise la naturalité du territoire canadien en 1972 dans son Manuel de planification du réseau des parcs nationaux. Inspiré du National Park System Plan états-unien, le manuel divise le Canada en 39 «régions naturelles» dont il définit les thèmes qui en «expriment l'essence même». Chaque région renvoie à une "catégorie géologique ou écologique» de «l'histoire naturelle» du Canada, laquelle exclut toute «valeur pittoresque [...], produit de la pensée humaine». Selon ce schéma, Forillon est situé dans la trentième région naturelle du pays, celle des monts NotreDame et Mégantic. Hors des frontières et de l'histoire des sociétés qui l'ont façonnée, la presqu'île devient exclusivement représentative des écosystèmes nationaux - par sa forêt boréale - et de la géologie canadienne - par ses forêts primitives du Dévonien ${ }^{78}$.

Depuis, dans le cas de Forillon, la production de la nature continue de servir la protection de la nation. Jusqu'au gouvernement formé en 2006 par Stephen Harper, le pouvoir fédéral défend, vis-à-vis du Québec en particulier, un «nationalisme d'attaque», une «réconciliation nationale»

73. Raymond Blake, «Intergovernmental Relations Trumps Social Policy Change: Trudeau, Constitutionalism, and Family Allowances", Journal of the Canadian Historical Association/Revue de la Société historique du Canada, 18, 1 (2007): 238.

74. "Les parcs nationaux. Déclaration au sujet de l'aménagement d'un parc à Pointe-Forillon», Débats de la Chambre des communes, $28^{\mathrm{e}}$ législature $1^{\mathrm{re}}$ session, 21 mai 1969, p. 8883-8885.

75. Extraite de la Bible, la maxime D'un océan à l'autre devient la devise nationale du Canada au lendemain de la Première Guerre mondiale et figure depuis sur les armoiries du Dominion.

76. MAINC, DPN, Compte-rendu des délibérations de l'audience publique tenue à Gaspé (Québec) le 17 novembre 1971, 1972, p. 1.

77. Eric Kaufmann, "Naturalizing the Nation": The Rise of Naturalistic Nationalism in the United States and Canada ", Comparative Studies in Society and History, 40, 4 (1998): 690.

78. MAINC, Manuel de planification du réseau des parcs nationaux, 1972, p. 48 et 95. Le Dévonien désigne le système géologique né il y a environ 420 millions d’années. 
puis un "nationalisme compétitif »" Le premier doit aboutir à l'endiguement du nationalisme québécois, le second, de 1981 jusqu'en 1995, à la prévalence d'une nation multiculturelle et pancanadienne, le troisième à la cohabitation entre le droit de l'État fédéral à affermir l'unité nationale et celui de chaque province à exister en tant que communauté politique singulière.

Au-delà de ces diverses façons de dire la nation subsiste en revanche une seule façon d'en faire la nature. Parcs Canada affine d'abord son argumentaire naturaliste. Avec la préservation de l'«intégrité écologique» pour mission première, l'administration entend transmettre «aux Canadiens des repères qui leur permettent $[. .$.$] de consolider leur unité nationale$ grâce à l'observation de la diversité des régions » et d'un paysage qui, "pendant des siècles $[\ldots]$ n'a subi $[\ldots]$ que l'action des agents naturels ». Définie en 1979, cette notion d'intégrité écologique se fait de plus en plus ouvertement nationaliste. Le ministre de l'Environnement Tom McMillan estima en 1985 que les parcs "peuvent aider à protéger l'intégrité de la souveraineté territoriale» et en 1988, la nouvelle loi sur les parcs nationaux donnait «la primauté [...] à la préservation de l’intégrité écologique » ${ }^{80}$.

Ainsi, à partir des années 1990, non seulement la nature est-elle écologisée, la nation tout entière l'est aussi. Au dire de l'administration fédérale, chaque parc "est un sanctuaire où la nature peut évoluer selon ses propres règles, comme elle le fait depuis la nuit des temps", et depuis peu au sein de «chacune des régions naturelles qui caractérisent notre pays et qui ont façonné notre histoire ${ }^{81}$. L'histoire récente, celle des hommes, pas celle de la nature, est ici insignifiante. Les provinces peuvent s'affronter sur la place à conférer aux héritages autochtones, français et britannique, les parcs continuent d’illustrer la canadianité. Au tournant des années 2000, considérant que les parcs "sont à notre image», l'administration canadienne va jusqu’à y légaliser l'objectif de «rétablissement de l’intégrité

79. Kenneth McRoberts, Un pays à refaire. L'échec des politiques constitutionnelles canadiennes (Montréal, Boréal, 1999), 67; Robert Bothwell, Ian Drummond et John English, Canada since 1945 : Power, Politics, and Provincialism (Toronto, University of Toronto Press, 1989 [2 éd.]), 438 («national reconciliation»); André Lecours et Daniel Béland, «Federalism and Fiscal Policy: The Politics of Equalization in Canada», Publius: The Journal of Federalism, 40, 4 (2010): 582 ("competitive nationalism»).

80. PC, Politique de Parcs Canada, 1979, p. 11 et 37; J. G. Nelson et R. C. Scace, dir., Patrimoine de demain (Ottawa, Environnement Canada, 1986), vol. 1, 275; «Loi modifiant la Loi sur les parcs nationaux et la Loi modifiant la Loi sur les parcs nationaux", Statuts du Canada, $33^{\mathrm{e}}$ législature $2^{\mathrm{e}}$ session chapitre 48,18 août 1988, p. 1229-1277.

81. PC, Projet de réseau des parcs nationaux, 1990, p. 3. 
écologique $»^{82}$. Associant dans le discours nature et nation, identité et canadianité, le pouvoir fédéral persiste donc à faire de la nature et de la géographie l'un des ciments de la «culture canadienne $e^{83}$ ».

Cette tentative d'écrire le récit d'une nation naturelle préside à l'histoire canadienne du parc québécois de Forillon. Accomplissant sa mission d'agence nationale, Parcs Canada le définit en 1978 comme un moyen de "prendre conscience de la valeur du patrimoine national», et en 2010 comme l'un de ces «lieux d'apprentissage merveilleux [qui] aident la population à mieux comprendre et apprécier le Canada ${ }^{84}$. Territoire national, Forillon est aussi un territoire naturel. Après avoir brûlé les habitations, réhabilité sa flore, protégé, déplacé et réintroduit sa faune, les responsables du parc s'efforcent de «maintenir [...] dans un état naturel [le] paysage [...] le plus spectaculaire à l'Est des Rocheuses ${ }^{85}$ ». Replacé dans un contexte québécois, lequel invite l'administration fédérale à la prudence quant à la valorisation des spécificités identitaires du territoire concerné, ce double processus de canadianisation de la nature et de naturalisation du Canada prend peut-être davantage de sens. Parcs Canada vante en 1975 Forillon et "ses 500 millions d'années d'histoire naturelle entremêlées à plus de 300 ans d'histoire humaine». Elle valorise en 1981 un "paysage harmonieux", au "passé très riche» mais dont «l'essence même fut mise en place au Siluro-dévonien, il y a 395 millions d'années». Et depuis la fin des années 1980, il entend «commémorer l'occupation humaine ${ }^{86}$. Aussi, la naturalité de la presqu'île l'emporte toujours davantage sur son humanité, sur les quatre millénaires de présence amérindienne, comme sur les quatre siècles de présence canadienne-française.

Depuis les années 1960, face à un mouvement trop souvent impulsé par le Québec, l'État fédéral peine à inventer le compromis qui ancrera les provinces, Québec en tête, dans l'imaginaire national canadien. Or, en réinventant la singularité historique d'une portion du territoire québécois, Parcs Canada peut substituer à l'histoire de la province celle, sans borne, de la nature canadienne: "terre d'accueil unique [qui] a façonné les humains qui y ont laissé leur empreinte, des Autochtones de la paléohis-

82. PC, Plan de réseau des parcs nationaux, 1997, «Préface», n.p.; «Loi concernant les parcs nationaux», Statuts du Canada, 36 législature $2^{\mathrm{e}}$ session chapitre 32, 20 octobre 2000, p. 5.

83. Robert Fulford, cité par E. Kaufmann, op. cit.: 689 ("geography sets the tone of Canadian culture»).

84. PC, Parc national Forillon: plan directeur, 1978, n.p.; PC, UGC, Parc national du Canada Forillon. Plan directeur, 2010, p. 111.

85. PC, Projet de réseau des parcs nationaux, 1990, p. 4.

86. PC, Parc national Forillon. Plan directeur, 1975, p. 35 ; PC, Secteur d'aménagement de Grande-Grève. Parc national Forillon, 1981, p. 3 ; PC, RQ, Amendement au plan de gestion. Parc national Forillon, 1988, p. 10, 24. 
toire aux derniers occupants avant la création du parc», Forillon «tel qu'il se révèlera à nos enfants» est le "témoin authentique» d'une "région naturelle» canadienne ${ }^{87}$. Tel est le récit patrimonial qu'a choisi l'État fédéral. Le temps du récit est celui d'une nature millénaire, son espace celui d'un territoire écologique national, et son lecteur le public supposé faire sien un territoire politiquement provincial et fédéral mais, surtout, naturellement canadien.

\section{DE LA VIOLENCE À L'INCOHÉRENCE PAYSAGÈRE}

Depuis 1970, les autorités provinciales puis fédérales monopolisent l'exercice de la violence concrète et symbolique afin d'imposer au groupe - les Gaspésiens - une conception particulière de leur identité - une relation harmonieuse et révolue avec l'environnement. Cette lutte paysagère aboutit néanmoins, aujourd'hui, à la réécriture du récit naturel et national. De prime abord, les résidents de la presqu'île paraissent victimes d'une violence concrète. Le gouvernement du Québec procède en juillet 1970 au dépôt du plan d'expropriation des 225 familles de Forillon et deux ans après, seul un couple de Rivière-au-Renard continue d'occuper sa propriété. Toutefois, le Tribunal de l'expropriation du Québec juge en 1973 que l'administration a profité de la méconnaissance des résidents pour acquérir leurs habitations à des prix inférieurs à ceux définis par la loi. Suivant ce jugement, le Procureur du citoyen octroie en 1975 à tous les expropriés une indemnisation de moitié supérieure à celle qui leur a déjà été versée ${ }^{88}$.

Parce qu'elles n'avaient d'autre choix que d'accepter les indemnisations qui entérinent leur expulsion ${ }^{89}$, les populations sont en fait davantage victimes d'une violence symbolique. En monnayant leur départ, elles autorisent l'administration provinciale soutenue par Parcs Canada à brûler leurs maisons. Ce faisant, elles valident la perspective selon laquelle leur présence nuit à l'intégrité écologique du territoire. Livrée par Lionel Bernier, avocat des expropriés et fils de l'un d'entre eux, la version romancée de l'expropriation donne la mesure de la douleur occasionnée.

Deux camions étaient stationnés dans l'entrée de sa maison. [...] À l'intérieur, M. Jeremy remplissait nerveusement sa valise. [...] Deux hommes empoignèrent sa valise $[\ldots]$ Il s'assit sur sa valise. [...] Il ne voyait plus rien... Était-ce la brume qui enveloppait tout? [...] Mais non, il n’y a pas de brume, M. Jeremy.

87. PC, UGC, Parc national du Canada Forillon. Plan directeur, 2010, p. 33.

88. Lionel Bernier, La bataille de Forillon (Montréal, Fides, 2001), 243-248.

89. Forillon, Québec, Entente générale de coopération Canada-Québec, 1970, p. 33. 
Il fait beau soleil. La brume, M. Jeremy, c'est juste dans vos yeux, dans votre tête. [...] Soudain, des flammes dansèrent dans sa brume. Ils venaient de mettre le feu à sa maison. [...] Il n'avait pas vu arriver son taxi. Quelqu'un le prit par le bras et le conduisit à l'auto. Dans un dernier sursaut, il tenta de s'échapper. Son chat était resté dans la maison... On le retînt. Des portières claquèrent. Il était déjà loin ${ }^{90}$.

Une fois converti ce milieu de vie quotidienne en un lieu de visites temporaires, Parcs Canada doit y faire respecter les «bons» usages et les «bonnes» représentations de la nature. Les populations locales refusant d'être exclues de l'espace écologique de la nation, derrière la surface harmonieuse du paysage se dessine cependant un paysage négocié.

En 1973, la fermeture de la route du Portage contraint les habitants de la presqu'île, pour aller de son nord à son sud, à en faire le tour complet. Ils ont beau protester - «nous ne faisons pas encore partie de la faune du parc», disent-ils en 1974 à l'administration -, rien n’y fait. Ils sont 1500 à envoyer une pétition en 1985 à la ministre de l'Environnement mais là encore, en vain. Pour Parcs Canada, «cette route [...] permet de maintenir la région qu'elle traverse dans la sauvagerie ${ }^{91}$ ». La situation est d'autant plus absurde aux yeux des Gaspésiens qu’en 1978, l'agence a accepté de rouvrir la route du Banc reliant, à l'est, les havres du Cap-des-Rosiers et de Cap-Bon-Ami : voulant offrir au public un aperçu de la vie «traditionnelle des pêcheurs», Parcs Canada ne pouvait courir le risque de voir les pêcheurs déserter ses havres ${ }^{92}$. D'ailleurs, à terme, seule cette pêche à quai et en mer est tolérée. «Autorisée lors de la création du parc surtout pour favoriser des relations de bon voisinage avec les résidents des villages touchés par l'expropriation", la pêche en ruisseaux est définitivement interdite en 1995. Quant à la pêche en eau profonde, Parcs Canada autorise depuis 1980 la «seule pêche artisanale» exercée selon des «méthodes traditionnelles $»^{93}$.

Face à cette obligation de tradition, les populations adoptent deux stratégies. Tandis qu'une minorité braconne ${ }^{94}$, une majorité s'escrime à

90. L. Bernier, op. cit., 203-204.

91. La Chambre de Commerce de l'Anse-au-Griffon, «Route 6: Route Panoramique, Parc Forillon», Le Soleil, dans PC-CSQ, dossier « Revue de presse 3671-F/ 4 », 4 janvier 1974, n.p.; William Fergus Lothian, Petite histoire des parcs nationaux du Canada, Ottawa, Environnement Canada. Parcs, 1987, 148.

92. MAINC, PC, Rapport préliminaire. Route panoramique. Parc national Forillon, 1978, n.p.

93. PC, Plan de conservation. Parc national Forillon, 1995, p. 37-38; PC, RQ, Plan de service. Les Havres et quais du parc national Forillon, 1980, n.p.

94. Les rapports d'activité font état d'environ, et en moyenne, dix cas annuels de chasse et de pêche «illégales». 
tirer profit des promesses de développement répétées par Parcs Canada. En 1985, les résidents de la presqu'île obtiennent l'organisation d'une séance de consultation publique. Il en ressort « une volonté manifeste [...] de faire en sorte que le parc Forillon devienne un élément moteur pour le développement économique ». Après s'être engagés à assumer un "rôle de "locomotive" touristique», les responsables du parc autorisent l'aménagement de pistes équestres, ouvrent deux havres de pêche au mouillage des plaisanciers et autorisent, à Penouille, l'offre d'un tourisme nautique ${ }^{95}$. Puis, à la fin des années 1990, ils doivent répondre à l'opinion selon laquelle «la préservation des éléments culturels et la mise en valeur de la présence humaine [...] ont été négligées». Aussi mettent-ils en valeur «l'intégrité de certains paysages culturels» et «la composition multiethnique» de la population: Micmac, Canadiens français et anglais deviennent les acteurs d'une seule "mosaïque culturelle $»^{96}$. Les expropriés sont d'ailleurs eux-mêmes intégrés à cette saga gaspésienne en 2010. Face à l'accusation publique d'un «désir [...] d'effacer l'histoire au profit de l'intégrité écologique", à Grande-Grave, photographies et objets d'époque de l'exposition «Ces Gaspésiens du bout du monde» commémorent cette "page d'histoire douloureuse» de Forillon ${ }^{97}$.

Ces compromis visent, d'une part, à mitiger le caractère polémique du parc Forillon. L'expropriation a beau incomber à l'État provincial, dans les mémoires québécoises l'État fédéral semble l'unique responsable. Le ministre québécois de l'Agriculture, Jean Garon, dénonçait en 1979 un "comportement sauvage, nazi» et trente ans plus tard, Le Soleil rappelle que «le gouvernement du Québec faisait la sale job du fédéral ${ }^{19}$ ». Voilà pourquoi en 2011, à la Chambre des communes, le gouvernement fédéral présente ses «excuses officielles» aux «familles expropriées». Le symbole est d'autant plus fort que le Québec ne s'excusera lui-même que huit mois plus tard99.

95. PC, RQ, Modifications proposées au plan de gestion du parc national Forillon, 1986, p. 8; PC, Amendement au plan de gestion. Parc national Forillon, 1988, p. 2-56.

96. PC, Parc national Forillon. Programme de consultation du public, 1994, p. 11; PC, La Gaspésie ethnoculturelle dans la péninsule de Forillon. Phase I, 2001, p. 16.

97. PC, Consultation publique pour la préparation du plan directeur. Compte-rendu, 2007, p. 13; Stéphane Marchand (directeur du parc), cité par Pierre Morel, "Pour ses 40 ans, un été fort occupé à Forillon", L'Avantage - Régional, dans PC-CSQ, dossier «Revue de presse 50G 3320-22 / F1 », 20 octobre 2010, n.p.

98. "Garon s'emporte en parlant de Mirabel et de Forillon", La Presse, dans PC-CSQ, ibid., 21 mai 1979, np.; Gilbert Lavoie, «Des larmes pour Forillon: à quand pour Rabaska?», Le Soleil, 21 octobre 2011, p. 3.

99. "Motion de l'opposition - Le Parc Forillon», Débats de la Chambre des communes, $40^{\mathrm{e}}$ législature $3^{\mathrm{e}}$ session vol. $145 \mathrm{n}^{\circ}$ 130, 14 février 2011, p. 8155-8156; "Motion sans préavis. Présenter des excuses officielles aux expropriés de Forillon ", Journal des Débats de l'Assemblée nationale, $39^{\mathrm{e}}$ législature $2^{\mathrm{e}}$ session, 42 , 52 (20 octobre 2011), 2960-2964. 
Les concessions faites par Parcs Canada renvoient, d'autre part, au caractère polysémique de Forillon. Si Parcs Canada considère agir au nom d'une harmonie passée entre l'homme et son milieu, pour beaucoup, l'agence défendait dans les années 1980 " "l’harmonie entre la terre et la mer"... sans l'homme» et continue, depuis, de mettre «l'accent sur l'intégrité écologique alors qu'il s'agit d'un paysage humanisé» ${ }^{100}$. Voilà pourquoi à Forillon, en 2010, dans le prolongement de la politique nationale de protection de l'«intégrité commémorative» des parcs légalisée en 1998, Parcs Canada déclare accorder autant d'importance à la protection de l'«intégrité culturelle» des lieux qu’à celle de leur «intégrité écologique»" ${ }^{101}$.

Les termes de cette négociation écologique, économique et culturelle participent plus largement du «fédéralisme d'ouverture » défendu depuis 2006 par le gouvernement Harper. Cette politique se traduit par la reconnaissance, aux "Premières Nations", d'un droit à l'autonomie et aux Québécois, d'un statut de "nation au sein d'un Canada uni » ${ }^{102}$. La nouvelle attitude des pouvoirs fédéraux envers les Autochtones est antérieure à l'arrivée au pouvoir de Harper. Mais à Forillon, c'est seulement sous son mandat que Parcs Canada effectue un "virage autochtone ${ }^{103}$ ", suivi d'un virage québécois. En créant un site micmac au cœur du parc en 2009, et en y commémorant en 2010 l'expropriation des Gaspésiens, les autorités espèrent articuler une territorialité nationale utopique - celle de l'intégrité écologique - avec une territorialité locale concrète - celle de l'intégrité culturelle.

Cette publicisation conjointe d'un espace à la fois vierge et anthropique signe l'incohérence, sinon l'échec, du paysage canadien au Québec en général, à Forillon en particulier. L'approche selon laquelle «l'intégrité écologique occupe trop de place» aux côtés d’une «intégrité culturelle» ${ }^{104}$ à réhabiliter y atteste d'une récente conversion à l'histoire. Prenant acte de l'existence d'autres communautés imaginées nationales et, surtout, de l'incapacité de la nature et de la nation canadiennes à en effacer les traces,

100. Jean-Marie Fallu, «L'histoire et la culture : les dimensions cachées de Forillon», Gaspésie, 23, 4 (1985): 6; J. M. Fallu cité par Gilles Gagné, «Les Gaspésiens envoient une volée de bois vert à la direction du parc Forillon», Le Soleil, dans PC-CSQ, dossier «Revue de presse 50G 3320-22/F1 », décembre 2006, p. 30 .

101. PC, Parc national du Canada Forillon. Plan directeur, 2010, p. 16.

102. Alain Noël, "Fédéralisme d'ouverture et pouvoir de dépenser au Canada», Revista d'Estudis Autonòmics i Federals, 7 (2008): 11.

103. Roch Samson, «Nature et culture dans les parcs nationaux. Reconnaître les liens », Revue d'histoire de l'Amérique française, 60, 1-2 (2006): 196.

104. PC, UGC, Parc national du Canada Forillon. Plan directeur, 2010, p. 16. 
ce «fédéralisme authentique» pourrait «sauvegarder l'unité du Canada»" Toutefois, Parcs Canada œuvre encore à la sauvegarde de l'intégrité écologique. Dans cette perspective, elle continue à faire des communautés autochtones et québécoises le produit passif de leur espace de vie, «cette terre d'accueil» qui aurait «façonné les humains» ${ }^{106}$. L'agence pourrait ainsi verser dans le "multinaturalisme» et le "multiculturalisme» ${ }^{107}$ afin de dissimuler, pour mieux la poursuivre, une logique fédérale nationale. Au-delà de ces deux interprétations du possible, la nature telle que les autorités fédérales cherchent à l'inventer au Québec révèle en tous les cas qu'aujourd'hui encore, "ce qu'est le Canada demeure un projet d'interrogation fastidieux, vraisemblablement insoluble ${ }^{108}$ ».

105. André Bernard, «Le système politique canadien et ses particularités », dans Alain Faure et Robert Griffiths, dir., La Société canadienne en débats. What Holds Canada Together? (Paris, L'Harmattan, 2008), 50.

106. PC, UGC, Parc national du Canada Forillon. Plan directeur, 2010, p. 33.

107. Catriona Mortimer-Sandilands, "The Cultural Politics of Ecological Integrity: Nature and Nation in Canada's National Parks, 1885-2000 ", International Journal of Canadian Studies/Revue internationale d'études canadiennes, 39-40 (2009): 174-178 ("multinaturalism», "multiculturalism»).

108. Bryan Palmer, Canada's 1960s. The Ironies of Identity in a Rebellious Era (Toronto, University of Toronto Press, 2009), 429 ("what Canada is remains a vexing, seemingly unanswerable, project of interrogation"). 